\title{
WILEY-VCH
}

\section{Insights into the crystallinity of layer-structured transition metal dichalcogenides on potassium ion battery performance: a case study of molybdenum disulfide}

\section{Yulian Dong, Yang Xu, * Wei Li, Qun Fu, Minghong Wu, * Eberhard Manske, Jörg Kröger and Yong Lei*}

\author{
Y. L. Dong, W. Li, Q. Fu, Prof. M. H. Wu \\ Institute of Nanochemistry and Nanobiology, School of Environmental and Chemical \\ Engineering, Shanghai University, Shanghai 200444, China \\ Email:mhwu@shu.edu.cn \\ Dr. Y. Xu, Prof. J. Kröger, Prof. Y. Lei \\ Institut für Physik, Technische Universität Ilmenau, Ilmenau 98693, Germany \\ Email: yang.xu@tu-ilmenau.de; yong.lei@tu-ilmenau.de
}

Prof. E. Manske

Institut für Prozessmess- und Sensortechnik, Technische Universität Ilmenau, Ilmenau 98693, Germany

Dr. Y. Xu

Department of Chemistry, University College London, London WC1H 0AJ, UK

Keywords: transition metal dichalcogenides, potassium ion batteries, crystallinity, rate capability, intercalation and conversion reactions

Layer-structured transition metal dichalcogenides (LS-TMDs) are being heavily studied in Kion batteries (KIBs) owing to their structural uniqueness and interesting electrochemical mechanisms. Synthetic methods are designed with primarily focusing on high capacities. The achieved performance is often the collective results of several contributing factors. It is important to decouple the factors and understand their functions individually. This work presents a study focusing on an individual factor, crystallinity, by taking $\mathrm{MoS}_{2}$ as a demonstrator. The performance of low- and high-crystallized $\mathrm{MoS}_{2}$ is compared and the results show the function of crystallinity is dependent on the electrochemical mechanism. Lower crystallinity can alleviate diffusional limitation in $0.5-3.0 \mathrm{~V}$, where intercalation reaction takes charge in storing K-ions. Higher crystallinity can ensure the structural stability of the $\mathrm{MoS}_{2}$ layers and promote surface charge storage in $0.01-3.0 \mathrm{~V}$, where conversion reaction mainly contributes. The low-crystallized $\mathrm{MoS}_{2}$ exhibits an intercalation capacity (118 mAh g$\left.{ }^{-1}\right)$, good cyclability ( $85 \%$ over 100 cycles) and great rate capability ( $41 \mathrm{mAh} \mathrm{g}^{-1}$ at $\left.2 \mathrm{~A} \mathrm{~g}^{-1}\right)$, and the high-crystallized $\mathrm{MoS}_{2}$ delivers a high capacity of $330 \mathrm{mAh} \mathrm{g}^{-1}$ at $1 \mathrm{~A} \mathrm{~g}^{-1}$ and retains $161 \mathrm{mAh}$ $\mathrm{g}^{-1}$ at $20 \mathrm{~A} \mathrm{~g}^{-1}$, being one of the best among the reported LS-TMDs in KIBs. 


\section{WILEY-VCH}

\section{Introduction}

Layer-structured transition metal dichalcogenides (LS-TMDs) have received world-wide attention in recent years. They are characterized by the weak van der Waals interaction between the layers and strong in-plane covalent bonding within the layers as well as the ultra-thinness of the layers. ${ }^{[1]}$ These features endow LS-TMDs with rich synthetic chemistry and favorable kinetics, making them heavily studied in many research domains. ${ }^{[2]}$ Among many emerging secondary battery technologies coming in spotlight, such as Li-S batteries, ${ }^{[3]} \mathrm{Li}^{-} \mathrm{O}_{2}$ batteries, ${ }^{[4]}$ and so on, ${ }^{[5]} \mathrm{K}$-ion batteries (KIBs) are considered to be a promising alternative of Li-ion batteries owing to the elemental abundance of $\mathrm{K}$, close electrochemical potential of $\mathrm{K}$ to $\mathrm{Li}$ in nonaqueous electrolytes, and higher ionic conductivity of $\mathrm{K}$ electrolytes relative to $\mathrm{Li}$ electrolytes. $^{[6]}$ The challenge to develop KIBs is that the large size of K-ions severely worsens the kinetics. The van der Waals gaps of LS-TMDs could serve as two-dimensional (2D) pathways and allow them to accommodate large-sized K-ions in the gaps without significant structural distortion, so they have attracted rapidly increasing attention as electrode materials of KIBs.

LS-TMDs store K-ions through a two-step electrochemical process that includes an intercalation reaction and a subsequent conversion reaction. The former uses van der Waals gaps to accommodate $\mathrm{K}$-ions and the latter causes the reduction of the metal upon continuous $\mathrm{K}$-ion insertion. Such kind of electrochemical mechanism generates a large K-ion storage capacity, mainly contributed by the conversion reaction at a low voltage range, and it has been a major motivation for the research of LS-TMDs in KIBs. Up to now, the reported LS-TMDs include sulfides ${ }^{[7]}$ and selenides, ${ }^{[8]}$ and some of them indeed have reached the state-of-the-art anode performance in KIBs. Various methods were applied to overcome the intrinsically deficient properties of LS-TMDs as electrode materials, so the reported performance could be achieved. Interlayer spacing engineering was used to expand the van der Waals gaps to facilitate K-ion diffusion. ${ }^{[7 b, 7 c, 8 b]}$ Phase engineering was used to modulate phase composition by 


\section{WILEY-VCH}

incorporating 1T-phase that has higher electronic conductivity than $2 \mathrm{H}$-phase. ${ }^{[9]}$ Making composites of LS-TMDs and carbon materials is a straightforward idea to increase the electronic conductivity of electrode matrix. ${ }^{[7 c-e, 8 b, 8 c]}$

Despite the applied methods have been proven effective to enhance LS-TMDs' performance, the enhancement is often the result of collective contributing factors, and it is difficult to decouple the factors and understand how exactly each of them contributes. For instance, expanding interlayer spacing not only causes regional atomic disorder ${ }^{[7 b]}$ but often induces the generation of 1T-phase. ${ }^{[10]}$ When compositing with carbon materials, on the one hand, interactions between the LS-TMD and the carbon, e.g., bonding and relative position, could greatly affect charge transport ${ }^{[7 \mathrm{~d}, 8 \mathrm{~b}]}$ and even expand interlayer spacing; ${ }^{[7 \mathrm{c}]}$ on the other hand, the carbon itself might be more decisive owing to the advantages of doping or porous structure. ${ }^{[7 \mathrm{e}, 7 \mathrm{~g}, 8 \mathrm{c}]}$ In order to understand individual factor as accurately as possible, two criteria should be met. First, one single model material is used, and its electrode matrix should be as simple as possible to avoid extra phases or components. Second, the synthetic method should be able to produce the control samples that possess same parameters to the maximum extent, except for the studying factor.

Herein, we present in this work a case study to demonstrate how the crystallinity of LSTMDs affects their KIB performance. $\mathrm{MoS}_{2}$ was chosen as the demonstrator because it is the most representative LS-TMDs and at the same time, the most studied of its kind in the field of KIBs. We used $\mathrm{MoS}_{2}$ as a single-phase and single-component anode without compositing with carbons, which meets the first criteria. We synthesized $\mathrm{MoS}_{2}$ nanosheet assemblies by a hydrothermal method and employed an annealing treatment to increase their crystallinity, which ensures other parameters remain unchanged and thus meets the second criteria. Our results show that lower crystallinity is beneficial in the voltage range $(0.5-3.0 \mathrm{~V})$ where only intercalation reaction contributes to K-ion storage, whereas higher crystallinity promotes high capacity and rate capability in the voltage range $(0.01-3.0 \mathrm{~V})$ where both intercalation and conversion 


\section{WILEY-VCH}

reactions occur. Moreover, the low-crystallized $\mathrm{MoS}_{2}$ delivered the best K-ion intercalation capacity, and the high-crystallized $\mathrm{MoS}_{2}$ exhibited one the best rate capability among all the reported LS-TMDs so far. With $\mathrm{MoS}_{2}$ being a representative LS-TMD and the two criteria being fully met, we believe our work could provide insights into K-ion storage in TMDs and may stimulate future study on viewing battery materials from different perspectives.

\section{Results and discussion}

$\mathrm{MoS}_{2}$ nanosheet assemblies were synthesized by a hydrothermal reaction at $220^{\circ} \mathrm{C}$ using $\mathrm{Na}_{2} \mathrm{MoO}_{4} \cdot 2 \mathrm{H}_{2} \mathrm{O}$ as Mo precursor and $\mathrm{CS}(\mathrm{NH})_{2}$ as $\mathrm{S}$ precursor. The low-crystallized sample obtained from the reaction is labeled as MS-220. After annealing at $700^{\circ} \mathrm{C}$ for $2 \mathrm{~h}$, highcrystallized sample was obtained and is labeled as MS-700. We first characterized the phase, surface chemistry and surface area/porous texture of the MS samples. Figure 1a shows two Xray diffraction (XRD) patterns that both can be indexed to $2 \mathrm{H}-\mathrm{MoS}_{2}$ with a hexagonal phase and a space group of P63/mmc (JCPDS 37-1492). A slight shift of the (002) peak from $14.4^{\circ}$ to $14.0^{\circ}$ as indicated by the dash line means the samples have an increased interlayer spacing of $0.63 \mathrm{~nm}$, which agrees with other reported nanosized $\mathrm{MoS}_{2 .}{ }^{[11]}$ Both samples show (100) and (110) peaks, which suggests the same atomic orientation along the basal planes. Compared to MS-220, MS-700 has narrower peak width and higher peak intensity as well as defined (103) and (105) peaks, proving that higher crystallinity and less disordered structure were obtained by the annealing treatment. Figure 1b shows the Raman spectra of the samples, where two strong peaks located at 379 and $406 \mathrm{~cm}^{-1}$ are assigned to the $2 \mathrm{H}-\mathrm{MoS}_{2}$ vibration modes of $\mathrm{E}^{1}{ }_{2 \mathrm{~g}}$ (in-plane) and $\mathrm{A}_{\mathrm{g}}^{1}$ (out-of-plane), respectively. ${ }^{[7 \mathrm{c}, 12]}$ No peaks of $1 \mathrm{~T}-\mathrm{MoS}_{2}$ was observed. The phase purity of the samples is further confirmed by the X-ray photoelectron spectroscopy (XPS) results. Survey spectra (Figure S1) show both samples contain only Mo and S. In the Mo 3d spectra (Figure 1c), the peaks at 228.7 and $231.9 \mathrm{eV}$ are attributed to Mo (IV) $3 d_{5 / 2}$ and $3 d_{3 / 2}$ of $2 \mathrm{H}-\mathrm{MoS}_{2}$, respectively. In the S 2p spectra (Figure 1d), the peaks at 161.5 and $162.7 \mathrm{eV}$ are 


\section{WILEY-VCH}

attributed to $S 2 p_{3 / 2}$ and $2 p_{1 / 2}$, respectively. All the peaks are symmetric, which indicates the sole existence of the $2 \mathrm{H}$ phase. Furthermore, Figure 1e shows the nitrogen adsorptiondesorption isotherms and the corresponding pore size distribution is highlighted in Figure 1f. The isotherms are somewhat ambiguous, most likely being type III isotherms. It is possible, however, that they are type IV isotherms with $\mathrm{H} 3$ hysteresis, as has been previously reported. ${ }^{[13]}$ The pore size distribution is similar between two samples and displays that the pore size is mainly in the range of 10-60 nm, which is ascribed to the space between the nanosheets, as will be seen in Figure 2. The specific surface areas of MS-220 and MS-700 are 9.94 and $10.46 \mathrm{~m}^{2}$ $\mathrm{g}^{-1}$, respectively, and the total pore volume is $0.08 \mathrm{~cm}^{3} \mathrm{~g}^{-1}$ for both samples. Therefore, the phase and surface characterizations reveal high similarities between MS-220 and MS-700, except for that the latter has higher crystallinity than the former. These results serve as an important base to compare the KIB performance of the samples.

We next characterized the morphology and micro-structures of the MS samples. Lowmagnification scanning electron microscopy (SEM) images (Figure S2) outline that both samples consist of high-yield hierarchical assemblies that are in a size of a few hundred nanometers. High-magnification SEM images (Figure 2a and 2b) show that the assemblies have a flower-like morphology formed by the interconnected nanosheets. The nanosheets have a typical thickness of $15 \mathrm{~nm}$ and a lateral size of around $100 \mathrm{~nm}$. There is no obvious morphological difference between the two samples. However, high-resolution transmission electron microscopy (HRTEM) measurement reveals the difference of their micro-structural difference. As shown in Figure 2c and 2d, both samples display a typical lamellar structure with an interlayer spacing of $0.63 \mathrm{~nm}$. MS-220 has more curvy edges that are caused by the discontinuous crystal fringes along the edges, as opposite to the straight fringes of MS-700. The high discontinuity is in accordance with the XRD results and suggests the lower crystallinity of MS-220 compared to MS-770. ${ }^{[10 a, 14]}$ SAED patterns (inset in Figure 2c and 2d) show the diffraction rings of (100), (103) and (110) planes, confirming the polycrystalline nature of both 


\section{WILEY-VCH}

samples. But clear diffraction spots can be observed in the pattern of MS-700, which can account for the higher crystallinity. The differed crystallinity can be further confirmed by the HRTEM images of the basal planes. As shown in Figure 2e and 2f, a lattice fringe of $0.27 \mathrm{~nm}$ can be observed in both samples and correspond to the spacing of the (100) plane, demonstrating the same atomic orientation along the basal dimension. However, the lattice fringes of MS-220 are not fully aligned over the entire area (Figure 2e), slightly rotating from one micro-area to another and showing discontinuity. In the case of MS-700 (Figure 2f), lattice fringes are well aligned with high continuity and minimum distortion, which can be evidenced by the less broadened (100) and (110) peaks in the XRD pattern (Figure 1a). Therefore, the morphology and micro-structure characterizations reveal the two samples have the same morphology, interlayer spacing, and atomic orientation of basal dimension. The only difference once again lies on the higher crystallinity of MS-700 over MS-220. These results serve as another important base to compare the KIB performance of the samples.

With the two bases established, we next investigated the effect of crystallinity on the MS samples' KIB performance. It is important to point out that $\mathrm{MoS}_{2}$ undergoes intercalation and conversion reactions to store alkali-metal ions, during which crystal reconstruction occurs when conversion reaction takes place at a low discharge voltage. ${ }^{[15]}$ Our previous study has showed that in the case of KIB, $\mathrm{K}$ intercalation occurs at a voltage above $0.5 \mathrm{~V}$ (vs. $\mathrm{K}^{+} / \mathrm{K}$ ), beyond which intercalation is replaced by conversion and the layered structure collapses. ${ }^{[7 b]}$ Thus, we tested the batteries in two voltage windows, $0.5-3.0 \mathrm{~V}$ and $0.01-3.0 \mathrm{~V}$. The performance was evaluated using cyclic voltammetry $(\mathrm{CV})$ and galvanostatic charge/discharge measurement at different rates.

KIB performance tested in 0.5-3.0 V is illustrated in Figure 3 and Figure S3. Different Kion storage behavior between the MS samples can be directly indicated by the CV results (Figure S3). For the case of MS-220, the first cathodic scan exhibits two broad peaks at 1.4 and $1.1 \mathrm{~V}$ as well as a slope extending to $0.01 \mathrm{~V}$, while the first anodic scan shows one broad peak 


\section{WILEY-VCH}

at $2.1 \mathrm{~V}$. All the peaks remain almost unchanged in the subsequent cycles except for the two cathodic peaks merge into a broader one. For the case of MS-700, a well-defined peak at $1.0 \mathrm{~V}$ and two peaks at 1.6 and $1.9 \mathrm{~V}$ can be found in the cathodic and anodic scans, respectively. Compared to MS-700, the obviously different cathodic scan of MS-220 is most likely due to its lower crystallinity and the more disordered structure, which may provide more intercalation sites than span over a wider range of voltage. ${ }^{[16]}$ As a result, the charge/discharge curves also display different features. MS-220 exhibits sloping curves with two small humps (1.4 and 1.1 V) during discharge and one $(2.1 \mathrm{~V})$ during charge (Figure 3a), whereas MS-770 exhibits a well-defined discharge plateau (1.1-1.3 V) and two merged semi-plateaus (1.6 and 1.9 V) during charge (Figure 3b). All these features agree well with their CV profiles. Besides the charge/discharge curves, what separates the two samples more significantly is the dependence of cycling capacities on rates. Cycling performance is shown in Figure 3c-3e and specifications are summarized in Table S1. MS-220 exhibits lower Cycle1 Columbic Efficiency (CE) than MS-700, presumably due to the more disordered structure and the existing defects that could trap K-ions in the deintercalation process. But the CEs of the two samples quickly become similar after initial cycles. Generally, both samples exhibit stable cyclability at all testing rates, with the Cycle100/Cycle2 capacity ratio being highest at $50 \mathrm{~mA} \mathrm{~g}^{-1}$, i.e., $85 \%$ for MS-220 $\left(99.3 / 117.5 \mathrm{mAh} \mathrm{g}^{-1}\right)$ and $83 \%$ for MS-700 (75.6/91.3 $\left.\mathrm{mAh} \mathrm{g}^{-1}\right)$. As far as we know, the obtained capacities and retention are the highest for K-ion intercalation storage in $\mathrm{MoS}_{2 \cdot}{ }^{[7 \mathrm{a}, 7 \mathrm{~b}]}$ We found that MS-220 delivered higher capacities than MS-700 at all rates, and the enhancement is higher at low rates than at high rates. We defined $\Delta C$ as the capacity difference between the two samples. As illustrated in Figure 3f, $\Delta C$ is around $25 \mathrm{mAh} \mathrm{g}^{-1}$ at $50 \mathrm{~mA} \mathrm{~g}^{-1}$, being averagely $30 \%$ of the capacity of MS-700, and it is close to zero at $1 \mathrm{~A} \mathrm{~g}^{-1}$. The MS samples were characterized after 50 cycles. The XRD patterns (Figure S4) clearly show the presence of the (002), (100) and (110) peaks, and the $\mathrm{E}^{1}{ }_{2 \mathrm{~g}}$ and $\mathrm{A}_{\mathrm{g}}^{1}$ peaks can be seen in the Raman spectra (Figure S5). Thus, these results demonstrate intercalation reaction dominates 


\section{WILEY-VCH}

the K-ion storage in the applied voltage window where the layered structure is preserved. Keeping in mind that the obtained intercalation capacities are typically below $120 \mathrm{mAh} \mathrm{g}^{-1}$, an enhancement by $30 \%$ unambiguously demonstrates a positive effect of the lower crystallinity of MS-220.

KIB performance was also tested in 0.01-3.0 V using the same procedure, and the results are shown in Figure 4 and Figure S6. At the voltage above $0.5 \mathrm{~V}$, each sample has same CV features (Figure S6) as those seen in Figure S3; at the voltage below 0.5 V, characteristically sharp peaks can be found near the cut-off voltage, which is attributed to the conversion reaction of $\mathrm{MoS}_{2}$ being reduced to $\mathrm{Mo}{ }^{[17]}$ The much higher current of the conversion peak relative to the intercalation peak suggests that conversion capacity contributes to the majority of the overall capacity of the samples. Indeed, as shown in the charge/discharge curves (Figure 4a and $4 \mathbf{b}$ ), long tails can be found below $0.5 \mathrm{~V}$ and contribute up to $400 \mathrm{mAh} \mathrm{g}^{-1}$ in the first discharge. MS-220 displays sloping curves over the entire voltage window, and the curves well overlap with each other, once again indicating the effect of its low-crystallized structure as discussed in the last paragraph. MS-700 displays a well-defined plateau at $0.2 \mathrm{~V}$, similar to other reported well-crystallized $\mathrm{MoS}_{2}$ in batteries. ${ }^{[18]}$ The XRD patterns and Raman spectra after cycles (Figure S4 and S5) further confirm that the layered structure collapses after repetitive conversion and crystal reconstruction. The samples were cycled at $50 \mathrm{~mA} \mathrm{~g}^{-1}$ (Figure 4c), $500 \mathrm{~mA} \mathrm{~g}^{-1}$ (Figure 4d) and $1 \mathrm{~A} \mathrm{~g}^{-1}$ (Figure 4e) for 100 cycles, and the specifications are summarized in Table S2. Although MS-700 exhibits higher Cycle1 CEs than MS-220, being similar to the results in $0.5-3.0 \mathrm{~V}$, the increase becomes less significant in $0.01-3.0 \mathrm{~V}$ because the conversion reaction replaces the intertercaltion reaction as the major contributer to the capacity, where repeating crystal structure restoration takes place and diminishes the negative effect of the disordered structure and defects on the initial CE. Surprisingly, the changing trend of the capacities is greatly different from what is observed in $0.5-3.0 \mathrm{~V}$. At a low rate of $50 \mathrm{~mA}$ $\mathrm{g}^{-1}$, MS-220 delivered capacities that are 110-140 $\mathrm{mAh} \mathrm{g}^{-1}$ higher than those of MS-700 (Figure 


\section{WILEY-VCH}

4f) over the cycles. But the capacities rapidly decay when increasing the rates, and they are much lower than those of MS-700 by averagely $105 \mathrm{mAh} \mathrm{g}^{-1}$ at $500 \mathrm{~mA} \mathrm{~g}^{-1}$ and $245 \mathrm{mAh} \mathrm{g}^{-1}$ at $1 \mathrm{~A} \mathrm{~g}^{-1}$. Comparing Figure 4f to Figure 3f, the sharp contrast highlights the importance of choosing the proper voltage window when cycling batteries and more importantly, how differently crystallinity of $\mathrm{MoS}_{2}$ affects its battery performance in a specific voltage window. It is worth pointing out that MS-700 exhibits impressive capacities and cycle stability at high rates. It delivered a Cycle2 capacity of $339.1 \mathrm{mAh} \mathrm{g}^{-1}$ at $500 \mathrm{~mA} \mathrm{~g}^{-1}$ and showed almost no decay after 100 cycles $\left(334.2 \mathrm{mAh} \mathrm{g}^{-1}\right)$. Even at $1 \mathrm{~A} \mathrm{~g} \mathrm{~g}^{-1}$, the Cycle100/Cycle2 capacity ratio is as high as $91 \%\left(305.5 / 333.9 \mathrm{mAh} \mathrm{g}^{-1}\right)$. To the best of our knowledge, the presented capacities and cycle stability at high rates are not only better than previously reported $\mathrm{MoS}_{2}$ in $\mathrm{KIBs},{ }^{[7 \mathrm{~d}, 7 \mathrm{e}]}$ but also comparable to the best results obtained in $\mathrm{VS}_{2}{ }^{[7 f]}$ and $\mathrm{VSe}_{2}{ }^{[8 \mathrm{a}]}$ among all the reported LS-TMDs in KIBs.

We have shown that when increasing rate, MS-220 tends to maintain a higher K-ion storage in 0.5-3.0 V while MS-700 tends to do it in 0.01-3.0 V. Our previous characterizations have shown the only difference between the samples is the crystallinity. Thus, we further sought to understand the effect of crystallinity on the observed performance. Figure $\mathbf{5}$ shows the CV curves recorded at the scan rates of $0.2-4 \mathrm{mV} \mathrm{s}^{-1}$ in the two voltage windows. In $0.5-3.0 \mathrm{~V}$, broad potassiation (reduction, labeled as Re) and depotassiation (oxidation, labeled as Ox) peaks are maintained at high scan rates for the case of MS-220 (Figure 5a). The peak positions remain almost unchanged, which signals very small polarization. For the case of MS-700 (Figure 5b), the Re-Ox peak separation becomes larger, meaning polarization becomes greater when increasing the scan rate. Better kinetic properties therefore can be found in MS-220. We used the relationship between peak current $(i)$ and scan rate $(v)$ to analyze the limiting factor of charge storage, according to the equation of $i=a v^{b}$. The $b$ value can be extracted from the slope by plotting $\log (i)$ against $\log (v)$, for which the value is 0.5 for a charge storage process controlled by semi-infinite linear diffusion while close to 1 for a surface charge storage process 


\section{WILEY-VCH}

free of diffusion control. ${ }^{[19]}$ As shown in Figure 5c, a good linear relationship can be seen in both samples. MS-220 has the $b$ values of $0.92(\mathrm{Re})$ and $0.85(\mathrm{Ox})$, which are higher than those of MS-700 (Re: 0.84; Ox: 0.81). This suggests that K-ion storage is less diffusion controlled in MS-220, which can be attributed to the lower crystallinity and more disordered structure. Such kind of structure could provide more K-ion intercalation sites and less K-ion diffusion resistance by forming percolation pathways via the opening of active diffusion channels, similar as reported in amorphous electrode materials. ${ }^{[20]}$ Given the fact that intercalation dominates the charge storage in $0.5-3.0 \mathrm{~V}$, it is no surprise to see that MS-220 maintains higher capacities than MS-700, particularly at high rates. We applied the same CV measurement and $b$ value analysis in $0.01-3.0 \mathrm{~V}$, and the results are in reverse, i.e., much steeper reduction peaks and higher polarization are seen in MS-220, while the peak positions of MS-700 remain almost unchanged with increasing the scan rate (Figure 5d and 5e). As a result, a better linear relationship can be found in MS-700 than in MS-220 (Figure 5f). MS-700 has higher $b$ values (Re: 0.96; Ox: 0.89) than MS-220 (Re: 0.78; Ox:0.83), meaning a more surface-dominated charge storage occurs in MS-700 at high rates. ${ }^{[6 b, 21]}$ As previously pointed out, conversion reaction contributes the majority of charge storage in $0.01-3.0 \mathrm{~V}$ and the multi-layered structure collapses during the reaction. As shown in Figure S7, the layers are exfoliated after cycles and display a curiver shape comparing to the pristine state, but the basal layers are well kept with a slightly expanded interlayer spacing of $0.65-0.66 \mathrm{~nm}$. Thus, high crystallinity can keep the structure of $\mathrm{MoS}_{2}$ basal layers intact after the layers are severely exfoliated, which facilitates the charge storage on the surface of the exfoliated layers, leading to high capacities at high rates.

Based on the above analysis, we cycled the MS samples at various rates to fully demonstrate their rate capability. MS-220 was cycled in 0.5-3.0 V and the results are shown in Figure 6a. It delivered reversible capacities of $108,86,72,60,48$ and $41 \mathrm{mAh} \mathrm{g}^{-1}$ at $0.05,0.1,0.2,0.5,1$ and $2 \mathrm{~A} \mathrm{~g}^{-1}$, respectively. A nearly full capacity recovery $\left(105 \mathrm{mAh} \mathrm{g}^{-1}\right)$ was obtained when reducing back to $0.05 \mathrm{~A} \mathrm{~g}^{-1}$. The obtained rate capability surpasses the reported results of $\mathrm{MoS}_{2}$ 


\section{WILEY-VCH}

tested in the intercalation voltage window. ${ }^{[7 a, 7 b]}$ MS-700 exhibited even more impressive rate capability in 0.01-3.0 V. As shown in Figure 6b, it delivered reversible capacities of 373, 350, $339,312,292,262$ and $228 \mathrm{mAh} \mathrm{g}^{-1}$ at $0.05,0.1,0.2,0.5,1,2$ and $5 \mathrm{~A} \mathrm{~g}^{-1}$, respectively. Even at rates as high as 10 and $20 \mathrm{~A} \mathrm{~g}^{-1}$, it retained capacities of 189 and $161 \mathrm{mAh} \mathrm{g}^{-1}$, respectively. When the rate was reduced to $0.05 \mathrm{~mA} \mathrm{~g}^{-1}$, a capacity of $\sim 400 \mathrm{mAh} \mathrm{g}^{-1}$ was recovered, which is even higher than the initial low-rate capacity. The charge/discharge curves can be found in Figure S8. We compared the obtained rate capability to previous reported results of LS-TMDs as KIB anodes, and MS-700 shows one of the best rate capabilities so far (Figure 6c). Moreover, the reported LS-TMDs were tested with a rate only up to $2 \mathrm{~A} \mathrm{~g}^{-1}$ and the incorporation of carbon materials into the electrode matrix, but MS-700 was tested at the rates far beyond $2 \mathrm{~A} \mathrm{~g}^{-1}$ and more importantly, retained high capacities at the high rates of 10 and $20 \mathrm{~A} \mathrm{~g}^{-1}$. The specifications of the capacities, cycling abilities, and rate capabilities are summerized in Table S3. In addition, the long-term cycling ability of MS-700 at high rates was examined by cycling it $5 \mathrm{~A} \mathrm{~g} \mathrm{~g}^{-1}$ for 300 cycles (Figure S9). It delivered a Cycle10 capacity of $215 \mathrm{mAh} \mathrm{g}^{-1}$ and retained 174 and $149 \mathrm{mAh} \mathrm{g}^{-1}$ after 100 and 300 cycles, respectively. Therefore, the presented electrochemical performance implies the potential of the MS samples as anode materials in KIBs.

\section{Conclusion}

In summary, we have shown by taking $\mathrm{MoS}_{2}$ as an example that not only does crystallinity of LS-TMDs significantly affect their K-ion storage, but also the effect is dependent on the storage mechanisms. When intercalation reaction takes control, lower crystallinity could alleviate the limitation of diffusion-controlled storage process by opening K-ion intercalation sites and diffusion channels. The low-crystallized $\mathrm{MoS}_{2}$ exhibited the highest intercalation capacity $\left(\sim 118 \mathrm{mAh} \mathrm{g}^{-1}\right)$, good cyclability ( $85 \%$ over 100 cycles), and best rate capability (41 $\mathrm{mAh} \mathrm{g}^{-1}$ at $\left.2 \mathrm{~A} \mathrm{~g}^{-1}\right)$. When intercalation and conversion reactions both contribute to store $\mathrm{K}$-ions, higher 


\section{WILEY-VCH}

crystallinity ensures the structural stability of the exfoliated $\mathrm{MoS}_{2}$ basal layers and promotes surface-controlled charge storage. The high-crystallized $\mathrm{MoS}_{2}$ delivered a high capacity of $\sim 330 \mathrm{mAh} \mathrm{g}^{-1}$ at $1 \mathrm{~A} \mathrm{~g}^{-1}$ and retained 189 and $161 \mathrm{mAh} \mathrm{g}^{-1}$ at 10 and $20 \mathrm{~A} \mathrm{~g}^{-1}$, respectively. The rate capability is not only among the best in all the LS-TMD anodes, but also obtained in the rate range well beyond the reported ones. LS-TMDs have rich structural chemistry and are expected to receive growing attention in the exciting research field of KIBs. We hope our work can shed some light on understanding the atomic structure-dependent KIB performance and offer insights for future material design in energy storage and conversion.

\section{Experimental Section}

Material preparation: low-crystallized $\mathrm{MoS}_{2}$ nanosheet assemblies were synthesized by a hydrothermal method. In a typical procedure, $0.3 \mathrm{~g} \mathrm{Na}_{2} \mathrm{MoO}_{4} \cdot 2 \mathrm{H}_{2} \mathrm{O}$ and $0.4 \mathrm{~g} \mathrm{CS}(\mathrm{NH})_{2}$ were dissolved in $30 \mathrm{ml} \mathrm{DI}$ water under stirring for $0.5 \mathrm{~h}$. Concentrated $\mathrm{HCl}$ was added to the solution drop-wise till the $\mathrm{pH}$ value changed to around 1 . The solution was kept stirring for another 0.5 $\mathrm{h}$, and then was transferred to a Teflon-lined stainless-steel autoclave. The autoclave was sealed and heated at $220^{\circ} \mathrm{C}$ for $24 \mathrm{~h}$. After naturally cooling to room temperature, the powders were collected by centrifuging, rinsed with DI water and ethanol for several times, and finally dried in vacuum at $60^{\circ} \mathrm{C}$. High-crystallized $\mathrm{MoS}_{2}$ nanosheet assemblies were obtained by annealing the low-crystallized $\mathrm{MoS}_{2}$ at $700^{\circ} \mathrm{C}$ for $2 \mathrm{~h}$ with a ramping rate of $5^{\circ} \mathrm{C} \min ^{-1}$.

Material characterizations: characterizations of the nanosheet assemblies were performed by X-ray diffractometry (D/MAX2500V PC diffractometer, $\mathrm{Cu} \mathrm{K} \alpha \lambda=1.54 \AA$ ), X-ray photoelectron spectroscopy (Thermo SCIENTIFIC ESCALAB 250Xi), Raman spectroscopy (inVia Raman microscope), nitrogen adsorption-desorption and pore size distribution (Micromeritics ASAP 2460), transmission electron microscopy (JEOL JEM-2100F) and scanning electron microscopy (Zeiss AURIGA $\left.{ }^{\circledR}\right)$. 


\section{WILEY-VCH}

Electrochemical measurements: working electrodes were fabricated by mixing the nanosheet assemblies, Super P and carboxymethyl cellulose sodium (CMC) with a weight ratio of 7:2:1. The mixture was uniformly coated (doctor-blade) on copper foils with a typical mass loading of $1-2 \mathrm{mg} \mathrm{cm}^{-2}$. It was then dried at $110^{\circ} \mathrm{C}$ under a vacuum for more than $12 \mathrm{~h}$. Electrochemical measurements were performed using the configuration of coin cells, CR2032, and the cells were assembled in an argon-filled glovebox with oxygen and moisture concentrations below $0.1 \mathrm{ppm}$. $\mathrm{K}$ disc as the counter electrode was separated from the working electrode by glass microfiber filter (Whatman, Grade GF/B). Electrolyte was 1 M potassium bis(fluorosulfonyl)imide (KFSI) in an ethylene carbonate (EC)/diethylene carbonate (DEC) solution (1:1). Galvanostatic charge/discharge was carried out on a Land CT 2001A battery testing system (Land, China) at rates of $0.05-20 \mathrm{~A} \mathrm{~g}^{-1}$ at room temperature. Cyclic voltammetry was carried out on a VSP electrochemical workstation (Bio-Logic, France) in a scan rate range of $0.1-4 \mathrm{mV} \mathrm{s}^{-1}$.

\section{Supporting Information}

Supporting Information is available from the Wiley Online Library or from the author.

\section{Acknowledgements}

This work was supported by the European Research Council (ThreeDsurface, 240144 and HiNaPc, 737616), German Research Foundation (DFG: LE2249/4-1 and LE2249/5-1), and National Natural Science Foundation of China (21577086).

Received: ((will be filled in by the editorial staff))

Revised: ((will be filled in by the editorial staff)) Published online: ((will be filled in by the editorial staff))

\section{References}

[1] a) C. Tan, X. Cao, X.-J. Wu, Q. He, J. Yang, X. Zhang, J. Chen, W. Zhao, S. Han, G.-H. Nam, Chem. Rev. 2017, 117, 6225; b) S. Manzeli, D. Ovchinnikov, D. Pasquier, O. V. Yazyev, A. Kis, Nat. Rev. Mater. 2017, 2, 17033. 


\section{WILEY-VCH}

[2] a) Y. Sun, S. Gao, F. Lei, Y. Xie, Chem. Soc. Rev. 2015, 44, 623; b) X. Chia, A. Y. S. Eng, A. Ambrosi, S. M. Tan, M. Pumera, Chem. Rev. 2015, 115, 11941; c) J. Xu, J. Zhang, W. Zhang, C. S. Lee, Adv. Energy Mater. 2017, 7, 1700571.

[3] a) W. Chen, T. Lei, W. Lv, Y. Hu, Y. Yan, Y. Jiao, W. He, Z. Li, C. Yan, J. Xiong, Adv. Mater. 2018, 30, 1804084; b) W. Chen, T. Lei, C. Wu, M. Deng, C. Gong, K. Hu, Y. Ma, L. Dai, W. Lv, W. He, X. Liu, J. Xiong, C. Yan, Adv. Energy Mater. 2018, 8, 1702348; c) W. Chen, T. Lei, T. Qian, W. Lv, W. He, C. Wu, X. Liu, J. Liu, B. Chen, C. Yan, J. Xiong, Adv. Energy Mater. 2018, 8, 1702889; d) T. Lei, W. Chen, W. Lv, J. Huang, J. Zhu, J. Chu, C. Yan, C. Wu, Y. Yan, W. He, J. Xiong, Y. Li, C. Yan, J. B. Goodenough, X. Duan, Joule 2018, 2, 2091; e) T. Lei, Y. Xie, X. Wang, S. Miao, J. Xiong, C. Yan, Small 2017, 13, 1701013.

[4] a) F. Meng, Z. Chang, J. Xu, X. Zhang, J. Yan, Mater. Horiz. 2018, 5, 298; b) P. Zhang, Y. Zhao, X. Zhang, Chem. Soc. Rev. 2018, 47, 2921; c) M. Zhou, Y. Xu, J. Xiang, C. Wang, L. Liang, L. Wen, Y. Fang, Y. Mi, Y. Lei, Adv. Energy Mater. 2016, 6, 1600448; d) H. Zhong, J. Wang, F. Meng, X. Zhang, Angew. Chem. Int. Ed. 2016, 55, 9937; e) L. Liang, Y. Xu, X. Wang, C. Wang, M. Zhou, Q. Fu, M. Wu, Y. Lei, J. Power Sources 2015, 294, 193.

[5] a) Y. Zhu, X. Yang, X. Zhang, Angew. Chem. Int. Ed. 2017, 56, 6378; b) Y. Zhu, Y. Yin, X. Yang, T. Sun, S. Wang, Y. Jiang, J. Yan, X. Zhang, Angew. Chem. Int. Ed. 2017, 56, 7881; c) T. Sun, Z. Li, H. Wang, D. Bao, F. Meng, X. Zhang, Angew. Chem. Int. Ed. 2016, $55,10662$.

[6] a) H. Kim, J. C. Kim, M. Bianchini, D. H. Seo, J. Rodriguez-Garcia, G. Ceder, Adv. Energy Mater. 2018, 8, 1702384; b) Y. Xu, C. Zhang, M. Zhou, Q. Fu, C. Zhao, M. Wu, Y. Lei, Nature Commun. 2018, 9, 1720; c) J. C. Pramudita, D. Sehrawat, D. Goonetilleke, N. Sharma, Adv. Energy Mater. 2017, 7, 1602911. 


\section{WILEY-VCH}

[7] a) X. Ren, Q. Zhao, W. D. McCulloch, Y. Wu, Nano Research 2017, 10, 1313; b) Y. Xu, F. Bahmani, M. Zhou, Y. Li, C. Zhang, F. Liang, S. H. Kazemi, U. Kaiser, G. Meng, Y. Lei, Nanoscale Horiz. 2019, 4, 202; c) K. Xie, K. Yuan, X. Li, W. Lu, C. Shen, C. Liang, R. Vajtai, P. Ajayan, B. Wei, Small 2017, 13, 1701471; d) B. Jia, Q. Yu, Y. Zhao, M. Qin, W. Wang, Z. Liu, C. Y. Lao, Y. Liu, H. Wu, Z. Zhang, Adv. Funct. Mater. 2018, 28, 1803409; e) B. Jia, Y. Zhao, M. Qin, W. Wang, Z. Liu, C.-Y. Lao, Q. Yu, Y. Liu, H. Wu, Z. Zhang, X. Qu, J. Mater. Chem. A 2018, 6, 11147; f) J. Zhou, L. Wang, M. Yang, J. Wu, F. Chen, W. Huang, N. Han, H. Ye, F. Zhao, Y. Li, Adv. Mater. 2017, 29, 1702061;

g) V. Lakshmi, Y. Chen, A. A. Mikhaylov, A. G. Medvedev, I. Sultana, M. M. Rahman, O. Lev, P. V. Prikhodchenko, A. M. Glushenkov, Chem. Commun. 2017, 53, 8272; h) M. Mao, C. Cui, M. Wu, M. Zhang, T. Gao, X. Fan, J. Chen, T. Wang, J. Ma, C. Wang, Nano Energy 2018, 45, 346.

[8] a) C. Yang, J. Feng, F. Lv, J. Zhou, C. Lin, K. Wang, Y. Zhang, Y. Yang, W. Wang, J. Li, S. Guo, Adv. Mater. 2018, 30, 1800036; b) W. Wang, B. Jiang, C. Qian, F. Lv, J. Feng, J. Zhou, K. Wang, C. Yang, Y. Yang, S. Guo, Adv. Mater. 2018, 30, 1801812; c) J. Ge, L. Fan, J. Wang, Q. Zhang, Z. Liu, E. Zhang, Q. Liu, X. Yu, B. Lu, Adv. Energy Mater. 2018, $8,1801477$.

[9] a) X. Geng, Y. Jiao, Y. Han, A. Mukhopadhyay, L. Yang, H. Zhu, Adv. Funct. Mater. 2017, 27, 1702998; b) M. Acerce, D. Voiry, M. Chhowalla, Nat. Nanotechnol. 2015, 10, 313.

[10] a) H. Dong, Y. Xu, C. Zhang, Y. Wu, M. Zhou, L. Liu, Y. Dong, Q. Fu, M. Wu, Y. Lei, Inorg. Chem. Front. 2018, 5, 3099; b) L. Huang, Q. Wei, X. Xu, C. Shi, X. Liu, L. Zhou, L. Mai, Phys. Chem. Chem. Phys. 2017, 19, 13696; c) Z.-T. Shi, W. Kang, J. Xu, Y.-W. Sun, M. Jiang, T.-W. Ng, H.-T. Xue, Y. Denis, W. Zhang, C.-S. Lee, Nano Energy 2016, $22,27$. 


\section{WILEY-VCH}

[11] a) J. B. Cook, H. S. Kim, Y. Yan, J. S. Ko, S. Robbennolt, B. Dunn, S. H. Tolbert, Adv. Energy Mater. 2016, 6, 1501937; b) X. Wang, X. Shen, Z. Wang, R. Yu, L. Chen, ACS Nano 2014, 8, 11394.

[12] T. S. Sahu, S. Mitra, Sci. Rep. 2015, 5, 12571.

[13] a) L. Zeng, C. Zheng, L. Xia, Y. Wang, M. Wei, J. Mater. Chem. A 2013, 1, 4293; b) Y. Xu, E. M. Lotfabad, H. Wang, B. Farbod, Z. Xu, A. Kohandehghan, D. Mitlin, Chem. Commun. 2013, 49, 8973.

[14] J. Xie, J. Zhang, S. Li, F. Grote, X. Zhang, H. Zhang, R. Wang, Y. Lei, B. Pan, Y. Xie, J. Am. Chem. Soc. 2013, 135, 17881.

[15] a) J. Park, J.-S. Kim, J.-W. Park, T.-H. Nam, K.-W. Kim, J.-H. Ahn, G. Wang, H.-J. Ahn, Electrochim. Acta 2013, 92, 427; b) Q. Li, Z. Yao, J. Wu, S. Mitra, S. Hao, T. S. Sahu, Y. Li, C. Wolverton, V. P. Dravid, Nano Energy 2017, 38, 342.

[16] H. Wang, D. Mitlin, J. Ding, Z. Li, K. Cui, J. Mater. Chem. A 2016, 4, 5149.

[17] J. Wang, C. Luo, T. Gao, A. Langrock, A. C. Mignerey, C. Wang, Small 2015, 11, 473.

[18] a) W. Ren, W. Zhou, H. Zhang, C. Cheng, ACS Appl. Mater. Interfaces 2016, 9, 487; b) X. Zhou, L.-J. Wan, Y.-G. Guo, Nanoscale 2012, 4, 5868.

[19] V. Augustyn, J. Come, M. A. Lowe, J. W. Kim, P.-L. Taberna, S. H. Tolbert, H. D. Abruña, P. Simon, B. Dunn, Nat. Mater. 2013, 12, 518.

[20] a) L. Fan, X. Li, B. Yan, J. Feng, D. Xiong, D. Li, L. Gu, Y. Wen, S. Lawes, X. Sun, Adv. Energy Mater. 2016, 6, 1502057; b) M. Zhou, Y. Xu, C. Wang, Q. Li, J. Xiang, L. Liang, M. Wu, H. Zhao, Y. Lei, Nano Energy 2017, 31, 514; c) Y. Xu, M. Zhou, C. Zhang, C. Wang, L. Liang, Y. Fang, M. Wu, L. Cheng, Y. Lei, Nano energy 2017, 38, 304.

[21] a) Z. Li, J. Ding, H. Wang, K. Cui, T. Stephenson, D. Karpuzov, D. Mitlin, Nano Energy 2015, 15, 369; b) H. Kim, Y.-U. Park, K.-Y. Park, H.-D. Lim, J. Hong, K. Kang, Nano Energy 2014, 4, 97. 


\section{WILEY-VCH}
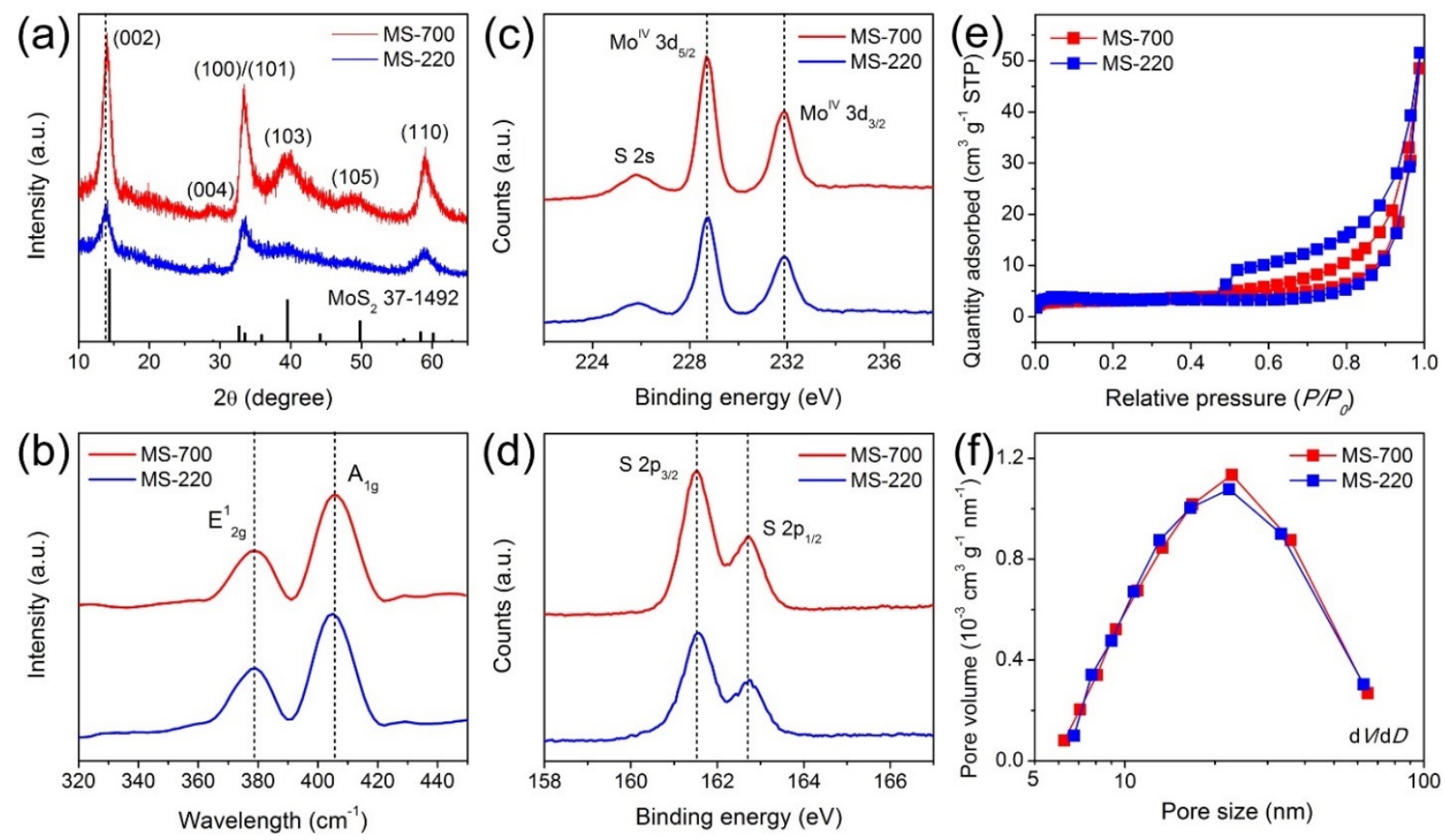

Figure 1. a) XRD patterns, b) Raman spectra, c) Mo 3d XPS spectra, d) S 2p XPS spectra, e) nitrogen adsorption-desorption isotherms, and f) pore size distribution of MS-220 and MS-700.
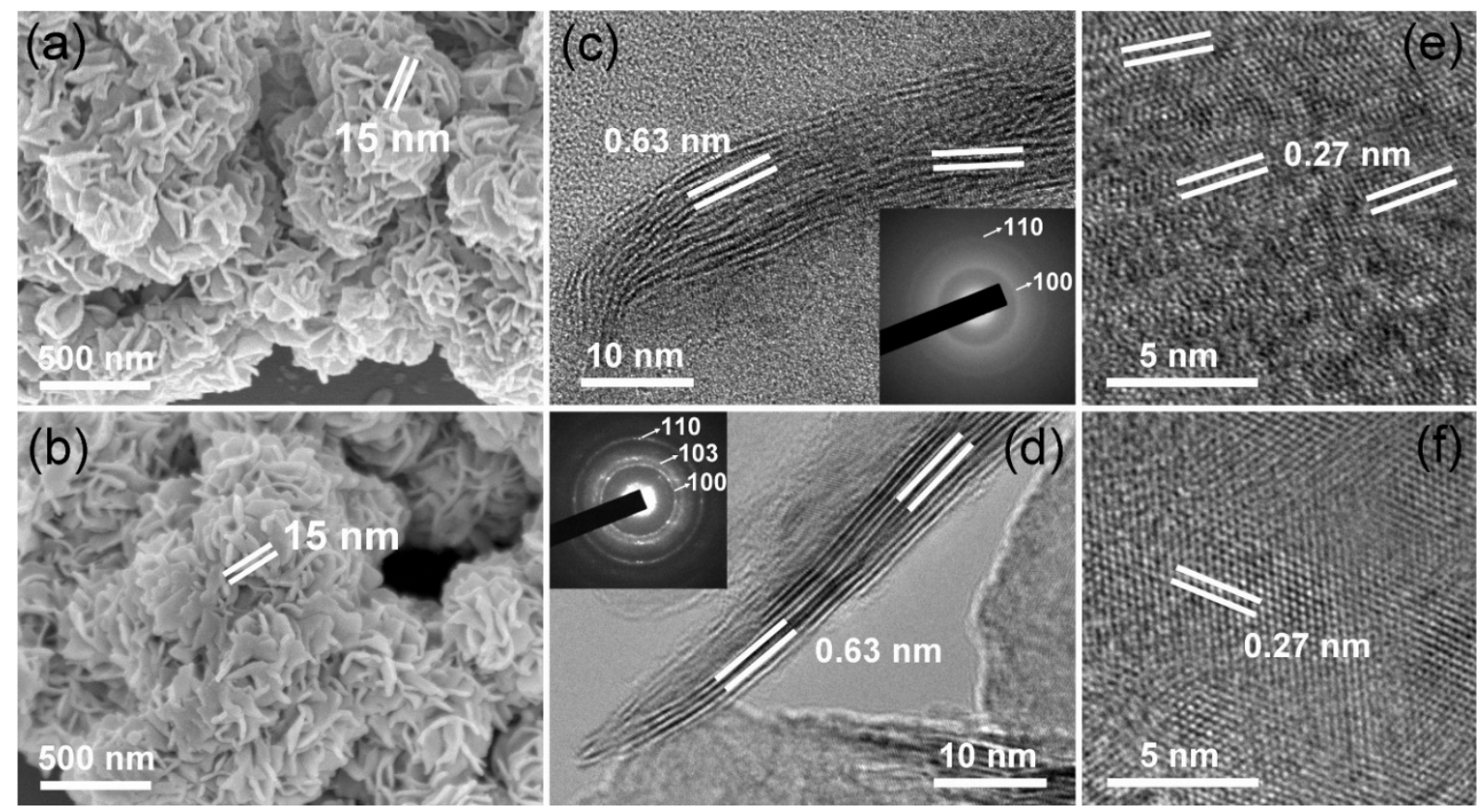

Figure 2. a,b) High-magnification SEM images, c,d) side-view HRTEM images, and e,f) HRTEM images of basal planes of a,c,e) MS-220 and b,d,f) MS-700. Insets in b,c) are SAED patterns. 
WILEY-VCH
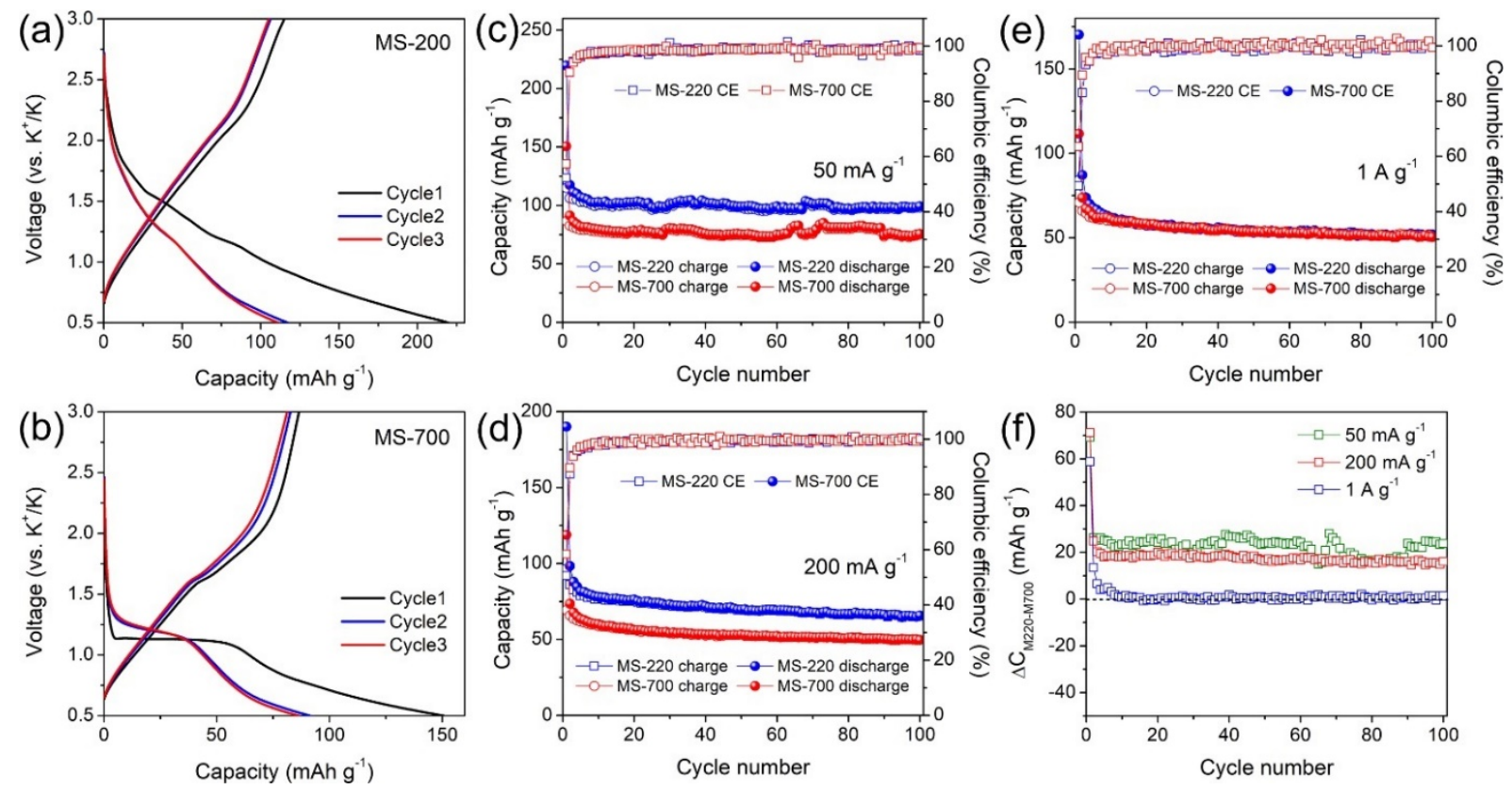

Figure 3. Charge/discharge curves of the first three cycles of a) MS-220 and b) MS-700 in 0.5-

$3.0 \mathrm{~V}$ at $50 \mathrm{~mA} \mathrm{~g}^{-1}$. Cycling performance at c) $50 \mathrm{~mA} \mathrm{~g}^{-1}$, d) $200 \mathrm{~mA} \mathrm{~g}^{-1}$ and e) $1 \mathrm{~A} \mathrm{~g}^{-1} . \mathrm{f}$ ) Capacity difference at each rate over cycles.
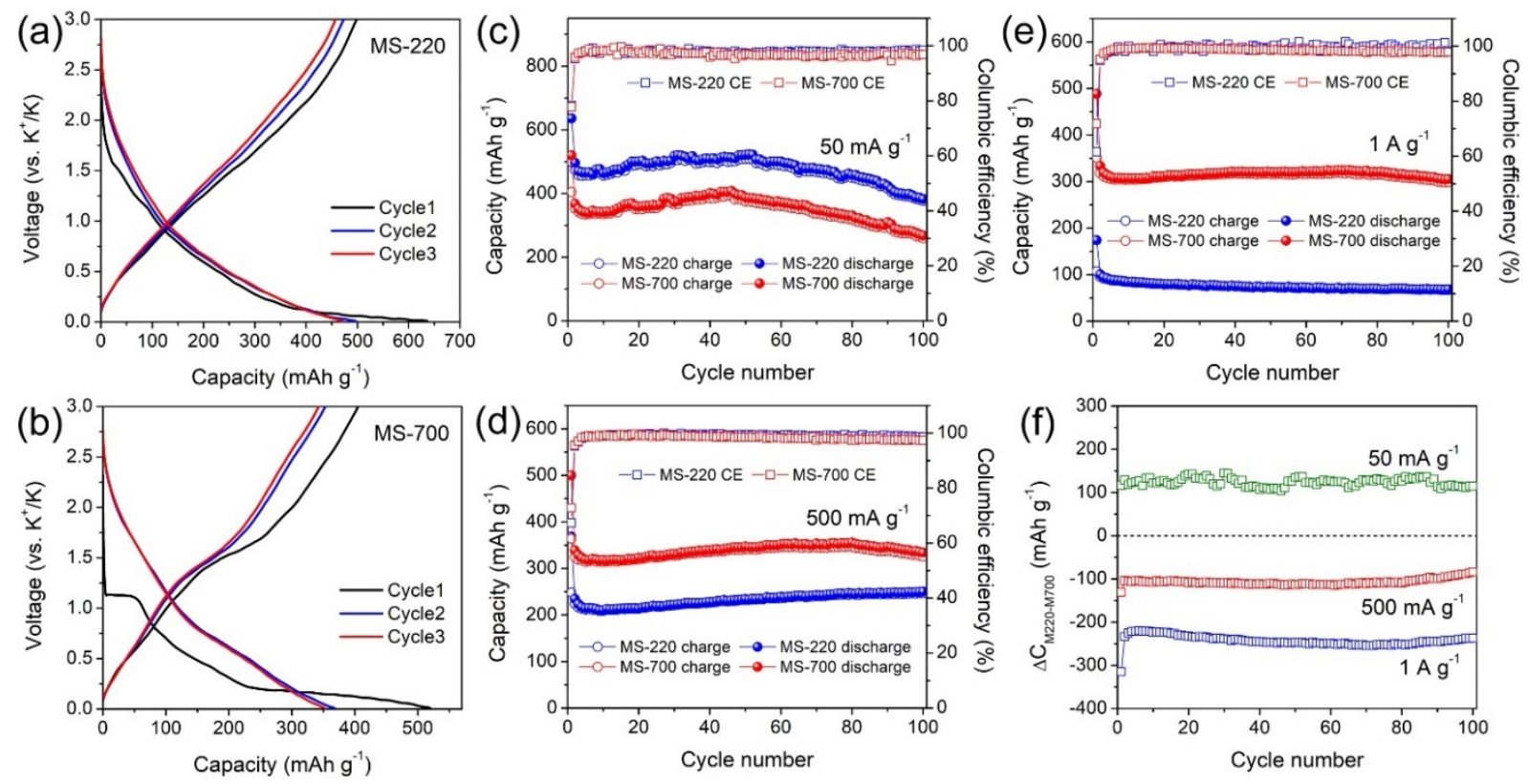

Figure 4. Charge/discharge curves of the first three cycles of a) MS-220 and b) MS-700 in 0.01-3.0 $\mathrm{V}$ at $50 \mathrm{~mA} \mathrm{~g}^{-1}$. Cycling performance at c) $50 \mathrm{~mA} \mathrm{~g}^{-1}$, d) $500 \mathrm{~mA} \mathrm{~g}^{-1}$ and e) $\left.1 \mathrm{~A} \mathrm{~g}^{-1} . \mathrm{f}\right)$ Capacity difference at each rate over cycles. 


\section{WILEY-VCH}
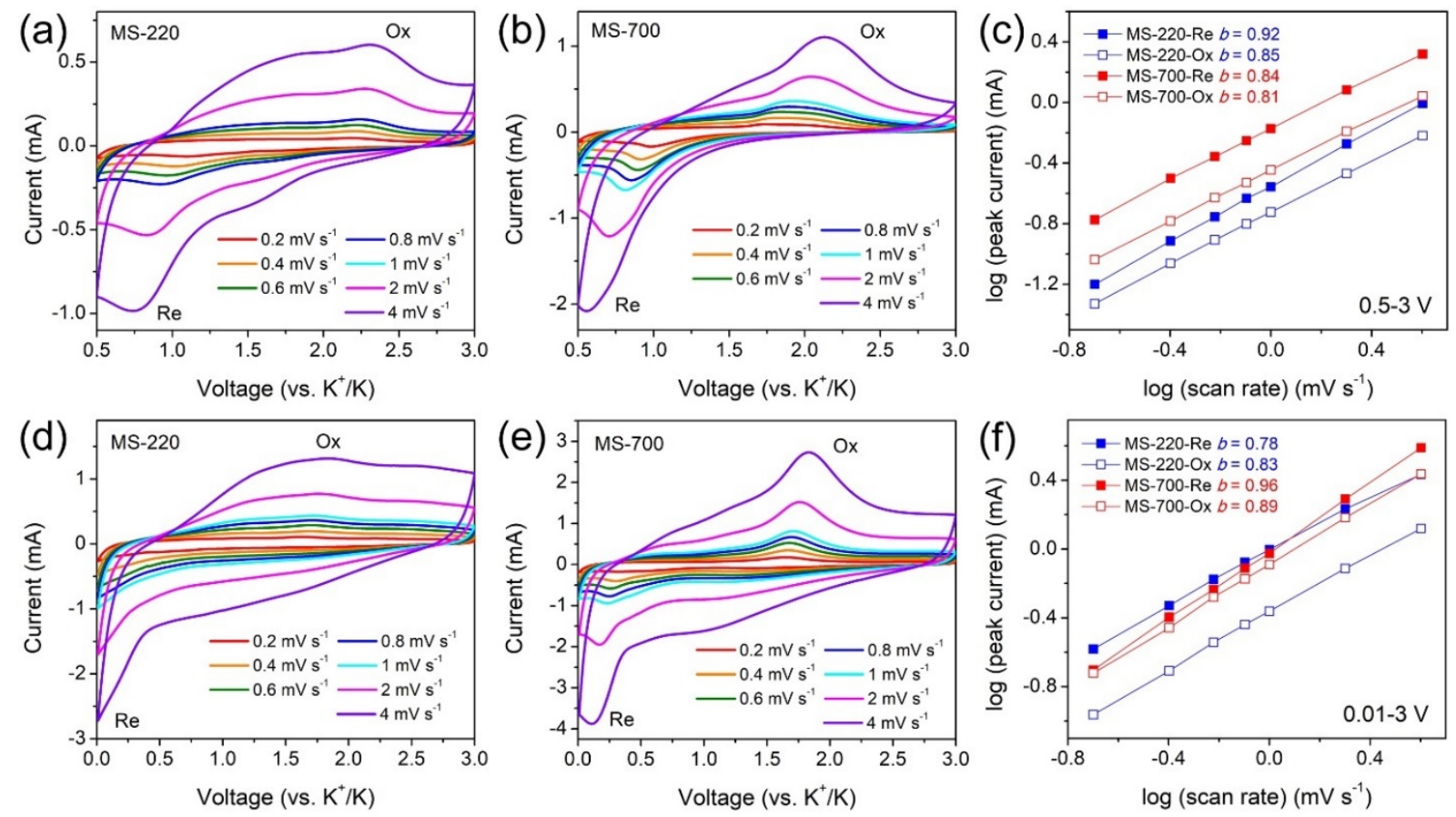

Figure 5. CV profiles of a,d) MS-220 and b,e) MS-700 in a,b) $0.5-3.0 \mathrm{~V}$ and d,e) $0.01-3.0 \mathrm{~V}$ at scan rates from 0.2 to $4 \mathrm{mV} \mathrm{s}^{-1}$. Determination of the $b$ values in c) $0.5-3.0 \mathrm{~V}$ and f) $0.01-3.0 \mathrm{~V}$.
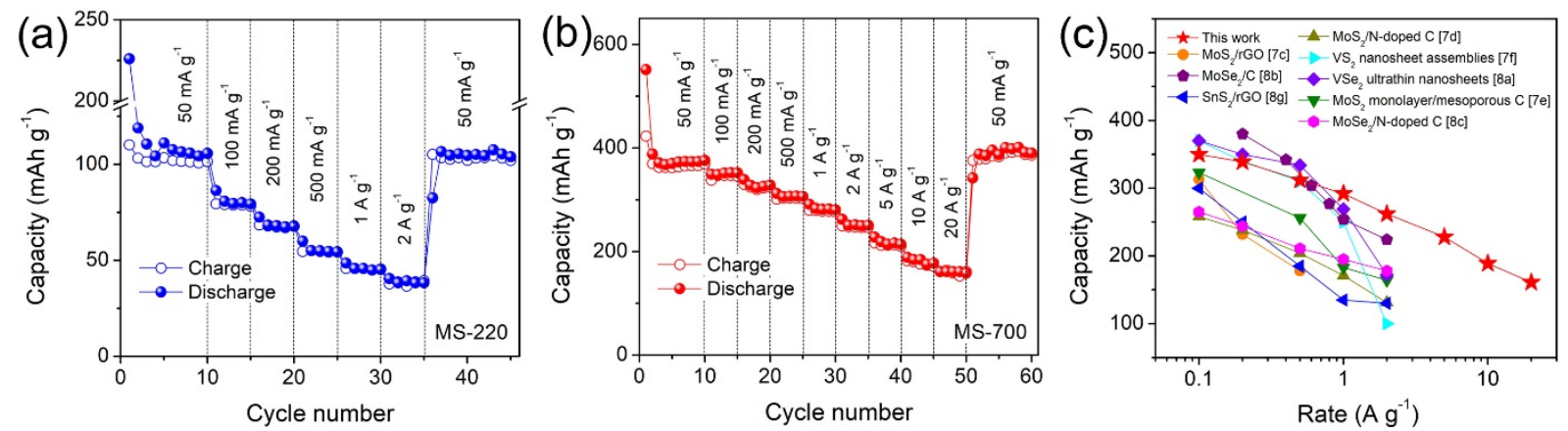

Figure 6. Rate capability of MS-220 in a) $0.5-3.0 \mathrm{~V}$ and MS-700 in b) $0.01-3.0 \mathrm{~V}$. c)

Comparison of the rate capability of MS-700 and previously reported LS-TMDs. 


\section{WILEY-VCH}

Crystallinity of layer-structured transition metal dichalcogenides is studied to reveal its effect on potassium ion battery performance, by taking molybdenum disulfide as a demonstrator. The effect is dependent on the $\mathrm{K}$-ion storage mechanism. Lower crystallinity benefits ion intercalation, while higher crystallinity promotes surface ion storage when conversion reaction mainly contributes to the capacity.

Transition metal dichalcogenides, potassium ion batteries, crystallinity, rate capability, intercalation and conversion reactions

Y. L. Dong, Y. Xu, * W. Li, Q. Fu, M. H. Wu, ${ }^{*}$ E. Manske, J. Kröger, Y. Lei*

Insights into the crystallinity of layer-structured transition metal dichalcogenides on potassium ion battery performance: a case study of molybdenum disulfide

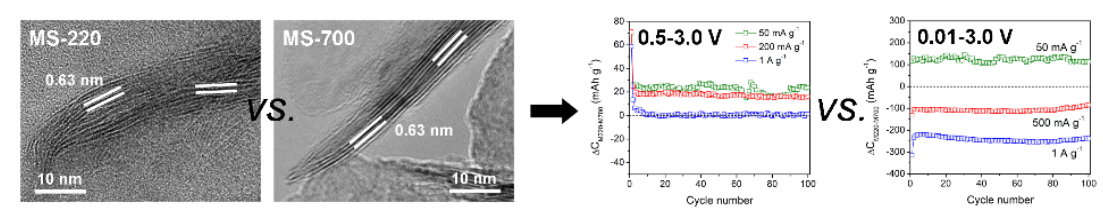

